\title{
Evaluating decontamination protocols for the isolation of Mycobacterium ulcerans from swabs
}

\author{
Enid Owusu ${ }^{1 *}$, Mercy J. Newman², Amos Akumwena², Elizabeth Bannerman² and Gerd Pluschke ${ }^{3}$
}

\begin{abstract}
Background: Mycobacterium ulcerans (M. ulcerans) is the causative agent of Buruli Ulcer (BU) disease. In order to inhibit the growth of the microbial contaminants during culture of $M$. ulcerans, it is necessary to decontaminate $\mathrm{BU}$ samples with effective chemical agents. This study aimed at investigating some selected chemicals as potential decontamination agents for the isolation of M. ulcerans from swabs.

Results: Povidone iodine at 0.5 and $1 \%$ exhibited the lowest contamination and recovery rate for microbial contaminants and M. ulcerans. The most effective decontamination method was the protocol using $2 \%$ cetylpyridinium chloride/4\% sodium chloride (recovery rate $=53 \%$, contamination rate $=14 \%$ ). The observed difference between the recovery rate of $2 \% \mathrm{CPC} / 4 \% \mathrm{NaC}$ and the other protocols was however not statistically significant $(p=0.76)$.

Conclusions: Two percent (2\%) cetylpyridinium chloride/4\% sodium chloride can be conveniently used as an alternative decontamination method for the isolation of $M$. ulcerans from swabs.
\end{abstract}

Keywords: Ghana, Buruli ulcer, Mycobacterium ulcerans, Decontamination, Cetyl pyridinium chloride, Oxalic acid

\section{Background}

Mycobacterium ulcerans (M. ulcerans) causes Buruli ulcer (BU) disease, the third most common Mycobacteriosis after tuberculosis and leprosy [1]. The disease has been reported in over 30 countries worldwide [2]. In Ghana, most endemic areas are located in water related rural farming communities, with limited access to healthcare. The associated deformities of patients as a result of the disease, coupled with the socio-economic effect on members within the affected communities makes it a public health issue [3]. Women and children under the age of 15 years are particularly affected [4]. Buruli ulcer disease manifests as skin nodules, papules, plaques, edema and ulcers. Currently, the BU disease is treated with rifampicin and streptomycin for an 8 week period [5]. Prior to the administration of the drugs however, the World Health Organization (WHO) strongly recommends that, suspected cases be confirmed by

\footnotetext{
* Correspondence: enidowusu15@yahoo.com; egowusu@ug.edu.gh ${ }^{1}$ Department of Medical Laboratory Sciences, School of Biomedical and Allied Health Sciences, University of Ghana, Accra, Ghana

Full list of author information is available at the end of the article
}

microbiological methods, including microscopy, culture, polymerase chain reaction (PCR) and histopathological analysis of diagnostic specimens from BU lesions [6].

Significant successes have been achieved with the management of BU disease since it became a global health challenge. This is as a result of intensive and extensive solution seeking investigations into a hitherto difficult situation. Investigations include (i) the extraction and use of mycolactone, the key virulent factor mediating Buruli ulcer disease for further research [7, 8], (ii) availability of information on drug susceptibility profiles of existing isolates to current antibiotics and potentially effective ones [9], (iii) M. ulcerans' viability in BU lesions post antibiotic treatment, indicative of treatment successes or failures, (iv) molecular epidemiology of the disease [10] and (v) disease surveillance. Most of these investigations have relied on M. ulcerans isolates from culture; a method for growing isolates of viable $M$. ulcerans in-vitro. This process therefore remains a very vital and important tool, for effective disease management. The culture process requires the use of diagnostic specimens from BU lesions. Faster growing microbial 
contaminants in BU lesions overgrow the M.ulcerans cultures, affecting their yield [11]. The reduction of these contaminants is usually achieved by processing the samples with chemical agents having selective antimicrobial properties in a process referred to as decontamination [12]. Effective decontamination protocols generally inhibit the growth of microbial contaminants with little or no inhibitory effect on viable $M$. ulcerans [13].

Commonly employed decontamination procedures in the primary isolation of $M$. ulcerans include sodium chloride (Petroff method) [14, 15], oxalic acid [14], N-acetyl cysteine $[15,16]$, Sulfuric Acid [16] (Buijtels PC1, Petit PL), benzalkonium chloride (BC) [17], trisodium Phosphate [15], sodium lauryl sulfate [18], and the equivalent 1- Hexadecylpyridinium chloride (HPC) methods [19-21]. To date, almost all decontamination protocols have their associated challenges $[22,23]$. These include higher toxicity to $M$. ulceren and slow processing time due to the reason that most protocols require a neutralization stage $[16,22]$.

Diagnostic specimens commonly used for $M$. ulcerans isolation include tissue samples, swabs and fine needle aspirates [10, 24]. Recovery from tissue specimens processed with $5 \%$ oxalic acid remains the highest reported decontamination process so far at $78 \%$ [25]. The effective use of antibiotics as definitive BU treatment has limited the availability of tissue samples [26], making swabs and fine needle aspirates more convenient alternatives. Decontamination protocols used for swabs should be relatively less harsh to bacilli in the already paucibacillary specimen. In addition, large open BU lesions tend to harbour spores of microbes including fungi and other hardy environmental microorganisms. To address these challenges, there is the need to design more decontamination protocols, not only with the sole objective of inhibiting growth of vegetative bacteria but also fungal contaminants [27]. Effective decontamination methods (especially chemical agents with known antifungal properties) can complement the commonly used $5 \%$ oxalic acid. The current study therefore investigated some chemicals as decontamination agents for the isolation of M. ulcerans from swabs.

\section{Methods}

Investigations and sample processing took place at the Bacteriology Department of the Noguchi Memorial Institute for Medical Research (NMIMR), University of Ghana, Legon, Tuberculosis Laboratory of the Public Health Reference Laboratory (PHRL), of the Ministry of Health and the chest clinic of the Korle-Bu Teaching Hospital (KBTH), Korle-Bu. This research was carried out between the periods June 2010 and April 2012.

Preparation of Buruli ulcer samples and clinical isolates Exudates from BU lesions of 35 subjects were swabbed for the study. Subjects were clinically suspected BU cases who reported at the Paakro and Asuboi health centers of the Eastern region of Ghana. The selected samples had been authenticated as being M. ulcerans positive by microscopy and IS2404 polymerase chain reaction (PCR). Specimen collection process involved a circular manipulation of the swab within the periphery of lesions [27].

Swabs in their receptacles were kept under cold storage and transported to the laboratory for analysis. Each swab specimen was eluted in $2 \mathrm{ml}(2 \mathrm{ml})$ of sterile phosphate buffered saline (PBS) in a $15 \mathrm{ml}$ capacity screw-capped test tube (3 mm diameter, Merck, Germany) [25].

Buruli ulcer sample for analysis was obtained as an eluted suspension, facilitated by vortexing swab tips with 10-15 glass beads for a minute. Bacterial clinical isolates used in the study included Pseudomonas aeruginosa, Salmonella typhi, Escherichia coli, Bacillus cereus, Staphylococcus epidermidis, Staphylococcus aureus and Klebsiella pneumonia. Aspergillus niger and Candida albicans were the only fungi isolates tested. Each microbial inoculum was prepared from a loopful of pure microbial culture emulsified in sterile distilled water to obtain turbidity comparable to McFarland's standard solution 0.5 , equivalents to $1 \times 10^{6}$ bacteria cells $/ \mathrm{ml}$.

\section{Chemical agents preparation}

Five (5) selected chemical agents, prepared at indicated working dilutions were used. These were (i) $0.5 \%$ povidone iodine (PI) [28], (ii) $1 \%$ povidone iodine (PI), (iii) $1 \%$ cetylpyridinium chloride/ $2 \%$ sodium chloride (iv) $2 \%$ cetyl-pyridinium chloride $/ 4 \%$ sodium chloride, (v) $5 \%$ oxalic acid (vi) $10 \%$ oxalic acid [29] (vii) $0.5 \%$ virkon and (viii) $1 \%$ virkon (ix) $20 \%$ benzalkonium chloride and (x) benzalkonium chloride at $40 \%$ [30,31]. Therefore, two dilutions each of the 5 chemical agents were prepared and used. Hence, 10 combinations were used for the subsequent process. Sterile distilled water was used as diluents for all chemical agents.

\section{Validating selected chemical agents as effective antimicrobials}

Each of the five chemical agents prepared in the dilutions indicated was mixed by vortexing with suspensions of microbial inoculums of the potential contaminants ( $P$. aeruginosa, S. typhi, E. coli, B. cereus, $S$. epidermidis, $S$. aureus, $K$. pneumonia, A. niger and $C$. albicans) in equal volumes of $200 \mu \mathrm{l}$ per test. Inoculums and chemical agents were vortexed for a minute and incubated at room temperature for $15 \mathrm{~min}$ with intermittent mixing. This process was aimed at ensuring even distribution and exposure. One Hundred microliters $(100 \mu \mathrm{l})$ of each test was inoculated onto Mueller-Hinton $(\mathrm{MH})$ agar plates and evenly spread to ensure uniformity after which the plates were incubated at $37^{\circ} \mathrm{C}$ for $24 \mathrm{~h}$. A 
test showing no growth was indicative of an effective inhibitory effect of the tested chemical agents at the prepared dilution.

\section{Inhibitory effect of chemical agents on microbial contaminants in BU samples}

Benzalkonium chloride was the only chemical agent that did not go through the validation stage successfully. Therefore, four successfully validated chemical agents were subsequently tested for their antimicrobial activity against microbial contaminants in BU samples. This implies that the number of chemical agents used for the study became 4 and in two dilutions each, making it 8 combinations. In brief, samples were obtained as swabbed exudates and pus from the periphery and crevices of the ulcers. Sample suspensions were obtained by agitating swab tips in fixed volume $(2 \mathrm{ml})$ of sterile phosphate buffered saline and glass beads. The suspension was then aseptically transferred to another sterile tube. For each of the suspensions an adjusted turbidity comparable to MacFarlands standard solution 1 was used. Chemical agents were mixed with BU sample suspensions in equal volumes of $400 \mu \mathrm{l}$. Tests were vortexed to ensure adequate exposure, and incubated at room temperature for $15 \mathrm{~min}$. One hundred microliters $(100 \mu \mathrm{l})$ of well mixed test was inoculated on Mueller Hinton agar plates and incubated at $37{ }^{\circ} \mathrm{C}$ for $24 \mathrm{~h}$. Media plates showing no growth was indicative of the antimicrobial effect of the chemical agent on the microbial contaminants in the BU samples.

\section{Examination of chemicals as effective decontamination agents}

The selected chemical agents at the specified dilutions were investigated for their potential as suitable decontamination agents for the recovery of $M$. ulcerans from swab specimens [32].

Two milliliters $(2 \mathrm{ml})$ aliquots of the sample homogenates were subjected to eight decontamination protocols using four chemical agents. The protocols were cetylpyridinium chloride at concentrations of 0.5 and $1 \%$, povidone iodine antiseptic at concentrations of 0.5 and $1 \%$, oxalic acid at concentrations of 5 and $10 \%$, and virkon disinfectant at concentrations of 0.5 and $1 \%$. Briefly, each of the test samples was incubated for 30 min under room temperature conditions after which they were centrifuged at $3000 \mathrm{rpm}$ for $15 \mathrm{~min}$. The supernatant was discarded and the pellet obtained re-suspended in $1.0 \mathrm{~mL}$ of sterile phosphate buffered saline (PBS) solution to obtain sample inoculums. Each sample inoculum $(100 \mu \mathrm{L})$ was seeded on Lowenstein-Jensen (L-J) slants in duplicates and gently spread over the surface. The tubes were loosely covered and examined daily until the media surface was completely dried. They were then tightly capped, arranged at a slanted angle of $30^{\circ}$ and incubated at $32{ }^{\circ} \mathrm{C}$. The cultures were examined weekly for the growth of contaminants and M. ulcerans (slowgrowers) for 12 weeks. The M. ulcerans colonies were identified by conventional laboratory methods [33] by subjecting the cultures with growth to Ziehl-Neelsen staining and a confirmatory IS2404 PCR [33]. Cultures showing no $M$. ulcerans growth after 12 weeks were considered negative, whilst those with growth were considered positive. Contaminated cultures were those exhibiting over $50 \%$ growth coverage of colonies other than that of $M$. ulcerans. Chemicals assessed as effective decontamination agents were the tests that exhibited high $M$. ulcerans recovery and low contamination rates. Controls for media, chemical agents and bacteria were set alongside all three experimental processes; testing the selected chemical agents (on test organisms) as effective antimicrobials, examination for their antimicrobial activity against microbial contaminants in BU samples and their potential as suitable decontamination agents for the recovery of $M$. ulcerans from swab specimens. They were incubated and read simultaneously with the tests at $32{ }^{\circ} \mathrm{C}$ [34].

\section{Data analyses}

Data from this study was stored in Microsoft excel (MS Excel) and analyzed with STATA 11 (Strata Corp, College Station, TX). Data analysis involved descriptions of estimated totals, arithmetic means, ranges and prevalence rates of the study variables. Association between the protocols was determined using the chi-square $\left(\chi^{2}\right)$ test, and based on $95 \%$ confidence interval. A P-Value less than 0.05 was considered statistically significant.

\section{Results}

Validation of selected chemicals as effective antimicrobial agents displayed various levels of activity against the test bacterial isolates (P. aeruginosa, S. typhi, E. coli, Bacillus cereus Staphylococcus epidermidis, Staphylococcus aureus, $K$. pneumoniae) and fungal isolates ( $A$. niger and $C$. albicans) (Table 1). All the test microbes were susceptible to both concentrations of povidone iodine (Table 1). E. coli, P. aeruginosa and S. typhi exhibited resistance to 1\% $\mathrm{CPC} / 2 \% \mathrm{NaCl}$ whilst Bacillus cereus and A. niger exhibited resistance to $5 \%$ oxalic acid. Virkon disinfectant at $0.5 \%$ exhibited antimicrobial activity against all except A. niger (Table 1). Meanwhile, 20\% BC (Timsen) and $40 \%$ BC (Timsen) had no antimicrobial activity against $P$. aeruginosa, $B$. cereus and $A$. niger.

Various degrees of growth inhibition on the samples were observed for the thirty five (35) BU samples (processed in duplicates to get a total of 70 samples and) tested with the selected chemical agents. Levels of inhibition which shows how many of plates were inhibited 
Table 1 Inhibitory effect of selected chemical agents on clinical isolates of Potential skin contaminants

\begin{tabular}{llll}
\hline Chemical agents & $\begin{array}{l}\text { In-vitro resistance of microbes } \\
\text { to chemical agents }^{\text {a }}\end{array}$ & $\begin{array}{l}\text { Test plates showing no } \\
\text { growth, } \mathrm{n}(\mathrm{N}=70)\end{array}$ & $\begin{array}{l}\text { Estimated activity levels } \\
\text { of chemical agents (\%) }\end{array}$ \\
\hline $0.5 \% \mathrm{Pl}$ & None of the microbes tested & 68 & 97 \\
$1 \% \mathrm{PI}$ & None of the microbes tested & 70 & 100 \\
$1 \% \mathrm{CPC} / 2 \% \mathrm{NaCl}$ & E. coli, P. aeruginosa \& S. typhi & 65 & 92 \\
$2 \% \mathrm{CPC} / 4 \% \mathrm{NaCl}$ & None of the microbes tested & 67 & 95 \\
$5 \%$ Oxalic acid & B. cereus \& A. niger & 64 & 91 \\
$10 \%$ Oxalic acid & None of the microbes tested & 67 & 96 \\
$0.5 \%$ Virkon & A. niger & 67 & 96 \\
$1 \%$ Virkon & None of the microbes tested & 67 & 96 \\
$20 \%$ BC (Timsen) & P. aeruginosa, B. cereus \& A. niger & 60 & 86 \\
$40 \%$ BC (Timsen) & P. aeruginosa \& A. niger & 63 & 90
\end{tabular}

PI Povidone iodine, CPC Cetyl pyridinium chloride, $\mathrm{NaCl}$ Sodium chloride, $B C$ Benzalkonium chloride

${ }^{a}$ Test microbes were P. aeruginosa, S. typhi, E. coli, Bacillus cereus, S. epidermidis, S. aureus, K. pneumonia, A. niger and C. albicans. \% $=\mathrm{n} / \mathrm{N} \times 100$

out of the 70 by a particular protocol ranged between $86 \%(60 / 70)$ and $100 \%(70 / 70)$. The highest levels of inhibition was exhibited by $0.5 \%$ povidone iodine and $1 \%$ povidone iodine; $97 \%$ (68/70 plates) and 100\% (70/70 plates) respectively. The activities of the chemical agents against the microbial sample contaminants were estimated based on the level of growth inhibition exhibited by test plates. The lowest level of $86 \%$ of antimicrobial activity was exhibited by $20 \%$ benzalkonium chloride (BC). Five percent oxalic acid exhibited an activity level of $91 \%$.

A summary of the effect of the eight decontamination protocols investigated against $35 \mathrm{BU}$ sample suspensions (prepared in duplicates) for the primary isolation of $M$. ulcerans from the BU samples is shown in Table 2. The recovery rates ranged between 0 and $53 \%$. The highest M. ulcerans' recovery rate was by $2 \% \mathrm{CPC} / 4 \% \mathrm{NaCl}$ at $53 \%$ whilst the lowest was by 0.5 and $1 \%$ povidone iodine (Table 2). The rate for $5 \%$ oxalic acid was $44 \%$. The secondary contamination rates of the M. ulcerans cultures were assessed for the various protocols. The least rate of secondary contamination was observed in all the two dilutions of povidone iodine (Table 2). The highest rate $(29 \%)$ of secondary contamination was recorded for the conventional (5\% oxalic acid) decontamination protocol followed by $1 \% \mathrm{CPC} / 2 \% \mathrm{NaCl}(21 \%)$ (Table 2).

\section{Discussion}

A major challenge in the primary isolation of $M$. ulcerans is contamination by fast growing microbes. Swab specimens, a more convenient diagnostic specimen tend to have lower yields due to limitations in sample collection. Optimization based studies aimed at complementing current decontamination protocols are essential. This study therefore investigated five chemicals for their effectiveness as decontamination agents in isolating $M$. ulcerans from swabs. Among the chemical agents investigated was the currently used $5 \%$ oxalic. The study was designed to preliminarily validate their effect on some likely bacterial and fungal skin contaminants. Among the bacterial contaminants were Bacillus cereus, E. coli, $K$. pneumoniae, $K$. species, P. aeruginosa, S.typhi, S. epidermidis and $S$. aureus. Candida albicans and

Table 2 Effect of protocols on the recovery of M. ulcerans from BU samples, in-vitro

\begin{tabular}{|c|c|c|c|c|}
\hline $\begin{array}{l}\text { Decontamination } \\
\text { protocols }\end{array}$ & $\begin{array}{l}\text { No. of tubes with M. ulcerans } \\
\text { growth per protocol }^{a}\end{array}$ & $\begin{array}{l}\text { Recovery rate (\%) } \\
\text { ( } p \text {-value) }\end{array}$ & $\begin{array}{l}\text { No. of tubes with } \\
\text { contamination }^{a}\end{array}$ & $\begin{array}{l}\text { Contamination rate }(\%) \\
\text { (p-value) }\end{array}$ \\
\hline $0.5 \% \mathrm{PI}$ & 0 & $0(0.00)$ & 0 & $0(0.00)$ \\
\hline $1 \% \mathrm{Pl}^{\mathrm{b}}$ & 0 & $0(0.00)$ & 0 & $0(0.00)$ \\
\hline $1 \% \mathrm{CP} / 2 \% \mathrm{NaCl}$ & 33 & $47(0.00)$ & 15 & $21(0.00)$ \\
\hline $2 \% \mathrm{CPC} / 4 \% \mathrm{NaCl}$ & 37 & $53(0.76)$ & 10 & $14(0.00)$ \\
\hline 5\% Oxalic acid & 31 & $44(0.07)$ & 20 & $29(0.00)$ \\
\hline 10\% Oxalic acid & 10 & $14(0.00)$ & 12 & $17(0.00)$ \\
\hline $0.5 \%$ Virkon & 36 & $51(0.40)$ & 12 & $17(0.00)$ \\
\hline $1 \%$ Virkon & 6 & $0.1(0.00)$ & 7 & $10(0.00)$ \\
\hline
\end{tabular}

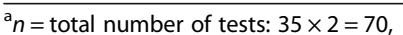

${ }^{\mathrm{b}}$ Conventional method, $B U$ Buruli ulcer, $P I$ Povidone iodine, CPC Cetyl pyridinium chloridem, NaCl Sodium chloride 
Aspergillus niger were the fungal contaminants used in the validation. Microbial contaminants isolated from BU samples collected for the study were also used in the validation process.

All tested chemical agents demonstrated varying degrees of inhibition against the isolates at their prepared dilutions. The effectiveness of povidone iodine and virkon as antimicrobial agents was clearly seen; as they exhibited the highest activity at 98 whilst $20 \%$ benzalkonium chloride (an antiseptic for the cleaning of diabetic wounds) showed the lowest activity at $86 \%$. In spite of high activity recorded by some of the chemical agents, $P$. aeruginosa and $A$. niger proved to be the most challenging to inhibit among all the microbial isolates tested. This finding brings to the fore the intrinsic resistance properties exhibited by $P$. eruginosa to most antimicrobials [11]. Similar observations were made by McClean et al. [11], where they also observed that $P$. aeruginosa was the predominant contaminant associated with the conventional isolation of Mycobacterium tuberculosis. That study also reported that $5 \%$ oxalic acid alone and in combination with $\mathrm{NaOH}$ was effective in eliminating $P$. aeruginosa [11]. The challenge in eliminating Aspergillus niger with the chemical agents used in the current study could be attributed to the fact that, most vegetative fungi and fungal spores are more resistant to decontamination agent. Biocide-microbial interaction usually occurs at the surface with little impact on the fungi, as few biocides are intended to focus on fungus cell as a major target.

The highest mean recovery of $M$. ulcerans from culture was exhibited by $2 \%$ cetyl pyridinium chloride $/ 4 \%$ sodium chloride (53.0\%) and $0.5 \%$ virkon $(51.0 \%)$ treated samples, and the lowest was observed in samples treated with 0.5 and $1 \%$ povidone iodine $(0 \%)$. The recovery rate of $1 \%$ cetyl pyridinium chloride $/ 2 \%$ sodium chloride and $5 \%$ oxalic acid were comparable at 44 and $41 \%$ respectively. This recovery rates are relatively less than 0.5 and $1 \%$ virkon disinfectant and $2 \% \mathrm{CPC} / 4 \% \mathrm{NaCl}$. In this study, M. ulcerans yield from $5 \%$ oxalic acid was comparatively lower at $41 \%$. This observation conforms to outcome from similar study conducted by Palomino and Portaels [14], where they observed a marked reduction in the growth rate of $M$. ulcerans in culture due to the harmful effect of oxalic acid on the culture of M. ulcerans from the BACTEC system [14]. Other investigators have however reported on high yields with the use of $5 \%$ oxalic acid. In a study by Yajko et al. [35], where they worked on fecal samples cultured on Lowenstein-Jensen media to evaluate various chemical decontamination protocols, they reported that the highest yield of Mycobacterium avium complex (MAC) was with oxalic acid [35]. Another study conducted in Ghana using 5\% oxalic acid gave an $M$. ulcerans isolation rate of $78 \%$ from tissue [25], and this is relatively higher compared to the $41 \%$ recovery rate observed for $5 \%$ oxalic acid from swabs.
The observed differences in the various studies including the current one appear to be due to various factors such as specimen types and treatment processes used, in addition to the array of selected chemical agents used for the evaluation studies. This study on the other hand, used swab specimens from open ulcers, cultured on Lowenstein-Jensen media. This confirms the variability of outcomes for recovery of $M$. ulcerans from culture, based on the specimens and the media used. The implication could be that whilst the decontamination step is very important for $M$. ulcerans' recovery, other factors could also affect the yield. In addition, it is possible that heavily contaminated specimens would require harsher treatments, but could also affect the viability of the target organism (M. ulcerans) in the sample.

Cetyl pyridinium chloride in sodium chloride though comparatively less harsh than povidone iodine, exhibited the best $M$. ulcerans recovery rate in this study. This phenomenon may be attributed to the antifungal properties of $\mathrm{CPC} / \mathrm{NaCl}[20,27]$ and its usefulness for the transport of specimens from peripheral centers to the reference laboratories for analysis, especially in underresourced endemic areas [19]. Anti-Fungal agents control the overgrowth of fungi, which characteristically spread at a rapid rate over the media surface. This implies that decontamination protocols incorporating anti-fungal properties will be required for effective decontamination.

This study has demonstrated that, single decontamination protocol only cannot improve the recovery of $M$. ulcerans from clinical specimens, especially swabs. In the culture of slow growing Mycobacterium, cultures are examined on a daily basis for 7 days for the presence of faster growing microbial contaminants. Even though povidone iodine was preliminarily validated as an effective antimicrobial agent against all contaminants tested, no colonies of $M$. ulcerans were observed. This outcome could be due to the fact that povidone iodine has a sterilizing effect in culture; property that made it unsuitable as a decontamination agents at the dilutions investigated in the current study. This observation corroborates the fact that, in investigating potentially novel decontamination protocols, a stepwise approach of validating its effect as a decontaminant at the specified dilution would be required. This would help reduce errors associated with the selection of appropriate decontamination protocols based on the premise that effective antimicrobial agents are by extension effective decontamination agents for the isolation of Mycobacterium. In addition, optimization of decontamination protocols for the isolation of Mycobacterium species would require the use of lower concentrations of the test agent and longer periods of exposure.

In a different study, Smithwick et al. [19] found 1\% cetyl pyridinium chloride/ $2 \%$ sodium chloride useful for 
the transport of sputum specimens from peripheral to reference laboratories. They observed that its effect was comparable to the conventionally used Nacetyl- L-cysteine. Results from this study corroborated his observation and also found it useful. Whilst the $1 \%$ povidone iodine protocol did not yield any $M$. ulcerans from culture, it recorded no contamination. This was also observed for $1 \%$ virkon disinfectant. Meanwhile, the highest rate (29\%) of secondary contamination was recorded for the conventional (5\% oxalic acid) protocol. Although this is a strong decontamination protocol, experimental period of exposure and low a concentration of agent in the culture medium may contribute to this contamination rate [11].

To the best of our knowledge, the use of povidone iodine and virkon as a decontamination agent in the primary isolation of Mycobacterium ulcerans in this study represents the first attempt to evaluate these chemical agents. The decontamination protocols utilizing the two concentrations of povidone iodine seems to affect not only the viability of the contaminating microbes, but it also inhibits the growth of the M. ulcerans in culture. This informs future projections where povidone iodine could be used at relatively lower concentrations to achieve more desirable results. Also, this study reports the effective use of $0.5 \%$ virkon, a broad spectrum disinfectant for the isolation of M. ulcerans from swabs.

\section{Conclusions}

Effective means of isolating $M$. ulcerans from diagnostic specimens, particularly swabs is required for control of BUD. This will inform policy on effective disease control measures targeted at reducing BU cases. The current research has demonstrated that, $2 \%$ cetylpyridinium chloride $/ 4 \%$ sodium chloride can be conveniently used as an alternative decontamination method for the isolation of $M$. ulcerans from swabs. This can contribute towards effective diagnosis and control of BU disease, worldwide.

\section{Abbreviations}

BC: Benzalkonium chloride; BU: Buruli ulcer; BUD: Buruli ulcer disease CPC/NaCl: Cetyl; CPC: Cetyl pyridinium chloride; GHS: Ghana Health Service; HPC: Hexa-decylpyridinium chloride; KBTH: Korle-Bu Teaching Hospital; M. ulcerans: Mycobacterium ulcerans; ml: millilitres; NaCl: Sodium chloride; NMIMR: Noguchi Memorial Institute for Medical Research; OA: Oxalic Acid; PBS: Phosphate buffered saline; PCR: Polymerase chain reaction; PI: Povidone iodine pyridinium chloride in Sodium chloride; WHO: World Health Organization
}

\section{Acknowledgements}

Authors wish to thank all the staff of the Bacteriology Department of the Noguchi Memorial Institute for Medical Research, University of Ghana, Legon, Chest clinic of the Korle-Bu Teaching Hospital and Tuberculosis Laboratory of the Public Health Reference Laboratory, Ministry of Health. These individuals are greatly appreciated for essential technical contribution; Mr. Emmanuel Ansah, Mr. Eric Aboga as well as Mr. Maxwell Quartey.

\section{Funding}

Funding for this research was provided by the DAAD (German Academic Exchange Programme).

\section{Availability of data and materials}

The majority of the data is included in the manuscript. All the other material used in the reported research shall be available only on request. The raw data is also available.

\section{Authors' contributions}

$E O, E B$ and MJN conceived and designed the experiments: $E O, A A$ and MJN participated in collection and analyses of the data and drafting of the manuscript. EO, MJN, and EB jointly contributed in the data interpretation and also developed the structure and arguments for the manuscript. MJN, GP and EO made critical revisions and approved final version. All authors read and approved the final manuscript.

\section{Competing interests}

The authors declare that they have no competing interests.

Consent for publication

Not applicable.

\section{Ethics approval and consent to participate}

This study was reviewed and approved by the Ethical and Protocol Review Committee of the Noguchi Memorial Institute for Medical Research (NMIMR) and the Ghana Health Service (GHS), Ghana. [Study \# 040/09-10].

\section{Author details}

'Department of Medical Laboratory Sciences, School of Biomedical and Allied Health Sciences, University of Ghana, Accra, Ghana. ${ }^{2}$ Department of Medical Microbiology, School of Biomedical and Allied Health Sciences, University of Ghana, Accra, Ghana. ${ }^{3}$ Swiss Tropical Institute, Basel, Switzerland.

Received: 22 February 2016 Accepted: 20 December 2016 Published online: 05 January 2017

\section{References}

1. Walsh DS, Portaels F, Meyers WM. Recent advances in leprosy and Buruli ulcer (Mycobacterium ulcerans infection). Curr Opin Infect Dis. 2010;23(5):445-55. doi:10.1097/QCO.0b013e32833c2209.

2. Thangaranj HS, Evans MRW, Wansbrough-Jones MH. Mycobacterium ulcerans disease; Buruli ulcer. Trans Royal Soc Trop Med and Hyg. 1999;93:337-40.

3. Aiga H, Amano T, Cairncross S, Adomako J, Nanas OK. Assessing waterrelated risk factors for Buruli ulcer: A case-control study in Ghana. Am J Trop Med Hyg. 2004;71:387-92.

4. Asiedu K, Etuaful S. Socioeconomic implications of Buruli ulcer in Ghana: a three year review. Am J Trop Med Hyg. 1998;59:1015-22.

5. World Health Organization. Provisional guidance on the role of specific antibiotics in the management of Mycobacterium ulcerans disease (Buruli ulcer). Geneva: WHO; 2004.

6. World Health Organization. Buruli ulcer. Diagnosis of Mycobacterium ulcerans disease. A manual for health care providers. Geneva: WHO/CDS/ CPE/GBUI/2001.4; 2001

7. Adusumilli S, Mve-Obiang A, Sparer T, Meyers W, Hayman J, Small PL. Mycobacterium ulcerans toxic macrolide, mycolactone modulates the host immune response and cellular location of M. ulcerans in vitro and in vivo. Cell Microbiol. 2005;7:1295-304.

8. Ortiz RH, Aguilar L, Estevez HO, Martin A, Herrera JL, Romo LF, et al. Differences in virulence and immune response induced in murine model by isolates of Mycobacterium ulcerans from different geographic areas. Clin Exp Immunol. 2009:157(2):271-81.

9. Marsollier L, Honore' N, Legras P, Manceau AL, Kouakou H, Carbonnelle B, et al. Isolation of three Mycobacterium ulcerans strains resistant to rifampin after experimental chemotherapy of mice. Antimicrob Agents Chemother. 2003:47:1228-32.

10. Walsh DS, Eyase F, Onyango D, Odindo A, Otieno W, Waitumbi JN, et al. Clinical and molecular evidence for case of buruli ulcer (M.ulcerans) in kenya. Am J Trop Med Hyg. 2009;81(6):1110-3. doi:10.4269/ajtmh.2009.09-0313.

11. McClean M, Stanley T, Stanley S, Maeda Y, Goldsmith CE, Shepard R, et al. Identification and characterization of breakthrough contaminants associated with the conventional isolation of Mycobacterium tuberculosis. J Med Microbiol. 2011;60:1292-8. 
12. Bratschi MW, Ruf M-T, Andreoli A, Minyem JC, Kerber S, Wantong FG, et al. Mycobacterium ulcerans Persistence at a Village Water Source of Buruli Ulcer Patients. PLoS Negl Trop Dis. 2014;8(3):e2756. doi:10.1371/journal.pntd.0002756.

13. Collins C, Grange J, Yates MD. Tuberculosis bacteriology (organization and practice) Reed Educational and professional Publishing Ltd UK, WHO/CDS/ CPE/GBUI/2001.4. 2nd ed. Geneva: The World Health Organization; 1997.

14. Palomino JC, Portaels F. Effects of decontamination methods and culture conditions on viability of Mycobacterium ulcerans in the BACTEC system. J Clin Microbiol. 1998;36:402-8.

15. Chatterjee M, Bhattacharya S, Karak K, Dastidar SG. Effects of different methods of decontamination for successful cultivation of Mycobacterium tuberculosis. Indian J Med Res. 2013;138(4):541-8.

16. Buijtels PC, Petit PL. Comparison of $\mathrm{NaOH}-\mathrm{N}$-acetyl cysteine and sulfuric acid decontamination methods for recovery of mycobacteria from clinical specimens. J Microbiol Methods. 2005;62(1):83-8.

17. Zabel LT, Albus J, Eiring P. Comparison of the effect of two decontamination procedures on the detection of Mycobacterium tuberculosis complex in respiratory specimens by a target-amplified test. Clin Microbiol Infect. 1998;4(2):116-8.

18. Manterola JM, Thornton CG, Padilla E, Lonca L, Corea I, Martinez E, Ausina V. Comparison of the sodium dodecyl sulfate-sodium hydroxide specimen processing method with the $\mathrm{C}_{18}$-carboxypropylbetaine specimen processing method using the MB/BacT liquid culture system. Eur J Clin Microbiol Infect Dis. 2003;22:35-42 [PubMed].

19. Smithwick RW, Stratigos CB, David HL. Use of cetylpyridinium chloride and sodium chloride for the decontamination of sputum specimens that are transported to the laboratory for the isolation of Mycobacterium tuberculosis. J Clin Microbiol. 1975;1:411-3.

20. Pardini M, Varaine F, Iona E, Arzumanian E, Checchi F, Oggioni MR, et al. Cetylpyridinium chloride is useful for isolation of Mycobacterium tuberculosis from sputa subjected to long-term storage. J Clin Microbiol. 2005:43:442-4

21. Corner LA, Trajstman AC. An evaluation of 1- Hexadecylpyridinium Chloride as a decontaminant in the primary isolation of Mycobacterium bovis from bovine lesions. Vet Microbiol. 1988;18:127-34.

22. Ambrosio SR, Oliveira EM, Rodriguez CAR, Neto FJS, Amaku M. Comparison of three decontamination methods for Mycobacterium bovis isolation. Braz J Microbiol. 2008;39:241-4. ISSN 1517-838224123.

23. Corner LA, Trajstman AC, Lund K. Determination of optimum concentration of decontaminants for primary isolation. New Zeal Vet J. 1995;43:129-33.

24. Cassisa V, Chauty A, Marion E, Ardant MF, Eyangoh S, Cottin J, et al. Use of Fine-Needle Aspiration for Diagnosis of Mycobacterium ulcerans Infection. J Clin Microbiol. 2010;48(6):2263-4. doi:10.1128/JCM.00558-10.

25. Yeboah-Manu D, Bodmer T, Mensah-Quainoo E, Owusu S, Ofori-Adjei D. Evaluation of decontamination methods and growth media for primary isolation of Mycobacterium ulcerans from surgical specimens. J Clin Microbiol. 2004;42:5875-6.

26. Cornet L, Richard-Kadio M, N'Guessen HA, Yapo P, Hossoko H, Dick R, et al Treatment of Buruli ulcers by excision-graft. Bull Soc Pathol Exot. 1992;85:355-8.

27. Phillips BJ, Kaplan W. Effect of cetylpyridinium chloride on pathogenic fungi and Nocardia asteroides in sputum. J Clin Microbiol. 1976;3:272-6.

28. Reimer K, Fleischer WA. Povidone-iodine in antisepsis-State of the art. Dermatology. 1997;195 Suppl 2:3-9.

29. Riemenschneider W, Tanifuji M. "Oxalic acid". in Ullmann's Encyclopedia of Industrial Chemistry. Weinheim: Wiley-VCH; 2002. doi:10.1002/14356007.a18_247.

30. Gasparini R, Pozzi T, Magnelli R, Fatighenti D, Giotti E, Poliseno G, et al. Evaluation of in vitro efficacy of the disinfectant Virkon. Eur J Epidermiology. 1995;11(2):193-7.

31. Hernandez A, Martro E, Matas L, Martin M, Ausina V. Assessment of in-vitro efficacy of $1 \%$ Virkon against bacteria, fungi, viruses and spores by means of AFNOR guidelines. J Hosp Infect. 2000;46(3):203-9.

32. Kent PT, Kubica G. Public health mycobacteriology: A guide for the level III laboratory. Atlanta: Centers for Disease Control; 1985

33. Herbinger $\mathrm{KH}$, Adjei $\mathrm{O}$, Awua-Boateng NY, Nienhuis WA, Kunaa L, Siegmund $\checkmark$, et al. Comparative study of the sensitivity of different diagnostic methods for the laboratory diagnosis of Buruli ulcer disease. Clin Infect Dis. 2009;48(8):1055-64. doi:10.1086/597398.
34. Grandjean L, Martin L, Gilman R, Valencia T, Herrera B, Quino W, et al. TB \& MDRTB testing by direct sputum culture in selective broth without decontamination or centrifugation. J Clin Microbiol. 2008;46:2339-44.

35. Yajko DM, Nassos PS, Sanders CA, Gonzalez PC, Reingold AL, Horsburgh Jr $C R$, et al. Comparison of four decontamination methods for recovery of Mycobacterium avium complex from stools. J Clin Microbiol. 1993;31:302-6.

\section{Submit your next manuscript to BioMed Central and we will help you at every step:}

- We accept pre-submission inquiries

- Our selector tool helps you to find the most relevant journal

- We provide round the clock customer support

- Convenient online submission

- Thorough peer review

- Inclusion in PubMed and all major indexing services

- Maximum visibility for your research

Submit your manuscript at www.biomedcentral.com/submit
Biomed Central 